\title{
ORIGINAL ARTICLE Event centrality as a unique predictor of posttraumatic stress symptoms and perceived disability following spinal cord injury
}

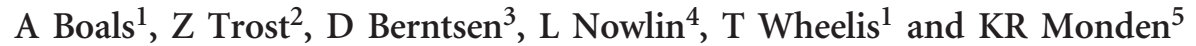

Study design: We conducted a cross-sectional study involving completion of self-report measures.

Objectives: Individuals who acquire a spinal cord injury ( $\mathrm{SCl}$ ) face numerous physical and psychological challenges, with the former receiving considerable less attention during the rehabilitation process. In this article, we examined event centrality as a unique predictor of psychological outcomes in a sample of individuals receiving rehabilitation for SCl. Event centrality refers to the extent to which individuals construe a stressful experience as a core part of their identity. In samples of individuals exposed to psychological traumas (for example, sexual assault or military combat), event centrality has emerged as a consistent and powerful predictor of posttraumatic stress symptoms (PTSSs). This is the first study to examine event centrality in an SCl sample.

Setting: Inpatient rehabilitation program in a large urban city in the Southwestern United States.

Methods: A sample of 55 participants in rehabilitation for a recent SCI completed measures of event centrality, PTSS, depressed mood and perceived disability.

Results: Event centrality was significantly related to perceived disability $(r=0.48)$ and PTSS $(r=0.31)$ and accounted for unique variance in these two outcomes after controlling for demographics and depressed mood.

Conclusion: Event centrality is common among individuals with $\mathrm{SCl}$ and may be a unique contributor to worse psychological and functional outcomes. We hope our findings will alert health-care professionals to the importance of event centrality.

Sponsorship: This study was supported by a grant from the Danish National Research Foundation (DNRF89).

Spinal Cord (2017) 55, 1023-1027; doi:10.1038/sc.2017.57; published online 30 May 2017

\section{INTRODUCTION}

Spinal cord injury (SCI) is a rare and sometimes devastating condition, with immense implications for the patient. The National Spinal Cord Injury Statistical Center (NSCISC) (2016) estimates there are 282000 people living with SCI in the United States, with up to 20000 new cases estimated to occur annually. Spinal cord trauma may result in various degrees of paralysis, loss of sensation and voluntary muscle control, reduced personal independence, and a decreased ability to participate in social and vocational activities. Individuals who acquire a SCI typically experience varied sequelae and chronic conditions, such as spasticity, pressure sores and chronic pain. ${ }^{1,2}$

Depression is perhaps the most prevalent psychological concern for individuals with SCI and, justifiably so, depression is the psychological outcome that has received the most research attention. However, given the threat to life and physical functioning, which often accompany SCI, it is perhaps not surprising to also observe elevated and sometimes clinical levels of posttraumatic stress symptoms (PTSSs) following SCI, a topic that has received considerably less research attention. Research on posttraumatic stress following SCI finds prevalence rates of $1-44 \%$ for posttraumatic symptoms. ${ }^{3-10}$ This wide disparity in prevalence rates mostly stems from variation in the amount of time-since-injury of the participant sample. Studies of participants with more recent SCIs (3-24 months post-injury) report prevalence rates between 14 and $44 \%,{ }^{3-5}$ whereas studies involving participants with more distant SCIs ( $>2$ years) report prevalence rates of $1-13 \%{ }^{6-8}$ However, it should be noted that one study did find a prevalence rate of only $1 \%$ in a sample of participants with a recent SCI. ${ }^{9}$ This study is one of the few studies to use a psychiatric interview to establish a diagnosis of posttraumatic stress disorder (PTSD), whereas most other similar studies describe the level of PTSS, without a formal diagnosis. Also of note, one study found a prevalence rate of $3.4 \%$ for participants whose time-since-SCI was 21 or more years. ${ }^{6}$ Such a finding demonstrates that some individuals continue to experience clinical levels of PTSSs for decades following the SCI. Although clinical levels of PTSS are less prevalent than clinical levels of depression following SCI, rates of PTSS following SCI are comparable to other types of trauma and constitute a clinical concern. ${ }^{5,10}$ PTSS typically reflect persistent re-experience of the trauma event, avoidance of trauma-relevant stimuli, general numbing of responsiveness and increased arousal. ${ }^{11}$ In addition to causing significant distress and social/functional impairment, PTSS can interfere with rehabilitation and long-term management of SCI. ${ }^{12}$ Research has examined a variety of sociodemographic, clinical and psychological factors that may contribute to development and maintenance of PTSS in this

${ }^{1}$ Department of Psychology, University of North Texas, Denton, TX, USA; ${ }^{2}$ University of Alabama at Birmingham, Birmingham, AL, USA; ${ }^{3}$ Aarhus University, Center on Autobiographical Memory Research, Aarhus, Denmark; ${ }^{4}$ Texas Scottish Rite Hospital for Children, Dallas, TX, USA and ${ }^{5}$ Craig Hospital, Denver, CO, USA Correspondence: Dr A Boals, Department of Psychology, University of North Texas, Box 311280, Denton, TX 76203, USA.

E-mail: adriel@unt.edu

Received 23 December 2016; revised 12 April 2017; accepted 12 April 2017; published online 30 May 2017 
Table 1 Sample size, means, s.d. and correlations between the outcome and predictor variables

\begin{tabular}{|c|c|c|c|c|c|c|c|c|c|c|}
\hline & $\mathrm{N}$ & $M$ (s.d.) & Range & 1 & 2 & 3 & 4 & 5 & 6 & 7 \\
\hline 2. PTSS & 54 & $0.79(1.19)$ & $0-4$ & & - & 0.12 & 0.05 & $0.39 * *$ & $-0.28^{*}$ & -0.07 \\
\hline 4. Pain intensity & 48 & $1.50(0.90)$ & $0-3$ & & & & - & $0.33^{*}$ & -0.13 & $0.30 *$ \\
\hline 5. Depressed mood & 55 & $5.09(4.62)$ & $0-21$ & & & & & - & -0.21 & 0.32 \\
\hline 6. Education & 54 & $7.11(2.31)$ & $1-12$ & & & & & & - & -0.01 \\
\hline
\end{tabular}

Abbreviation: PTSS, posttraumatic stress symptom.

Gender was coded $0=$ male and $1=$ female. Correlations with gender were point biserial correlations.

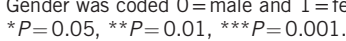

population. ${ }^{8}$ Characterizing potential risk factors for PTSS in SCI is critical to facilitate efforts at prevention and appropriate management/ intervention.

In the larger trauma literature, the construct of 'event centrality' has emerged as a consistent and powerful predictor of posttraumatic symptomatology following a stressful/ traumatic life event. ${ }^{13,14}$ Event centrality refers to the extent to which an individual perceives the event as a central part of his/her identity. It is composed of three functions of an autobiographical memory of a stressful event: the extent to which the event becomes (1) a reference point for everyday inferences, (2) a turning point in the life story and (3) a core component of personal identity. Acquiring a serious injury or disability-associated condition may comprise such an event. Subsequently, these features result in a wide range of stimuli becoming capable of triggering thoughts and emotions regarding one's condition. Indeed, greater levels of event centrality have been found to be associated with posttraumatic symptomatology in a broad range of populations, including war veterans, ${ }^{15}$ both physical and sexual assault victims, ${ }^{16,17}$ as well as college-age participants ${ }^{13,18}$ and older adults. ${ }^{19}$ Across populations, event centrality has remained a unique predictor of variance in PTSS after controlling for anxiety, depressed mood, dissociation, as well as other known predictors. ${ }^{13,14,20,21}$ In addition to cross-sectional evidence, event centrality has been shown to predict PTSS in longitudinal and prospective studies following stressful/traumatic life events. ${ }^{22-24}$

Although event centrality is typically in reference to a specific event, such as a sexual assault or natural disaster, recent research has similarly construed event centrality in reference to a persistent physical or health condition. For example, Perri and Keefe ${ }^{25}$ examined the impact of event centrality in a sample of patients with persistent pain. Participants completed a measure of event centrality in reference to 'the experience of persistent pain' ( $p$ 266). The results revealed higher levels of event centrality with respect to chronic pain were significantly related to greater pain intensity, life interference because of pain and psychological distress.

Given that SCI is a tremendously disruptive life event that often calls for major adjustments across several life domains, it is plausible that event centrality appraisals may affect psychosocial outcomes (in particular, PTSS) among individuals who sustain such injury. The current cross-sectional study represents the first effort to examine event centrality among individuals who have sustained an SCI. The aims of this study were therefore to: (1) characterize levels of event centrality in a sample of individuals receiving inpatient rehabilitation following SCI, (2) examine associations between event centrality and report of PTSS, (3) examine associations between event centrality and other SCI-relevant outcomes, including pain intensity, perceived disability and depressed mood and (4) examine the unique contribution of event centrality to PTSS and other outcomes. We hypothesized that magnitude of event centrality would significantly account for variance in outcome measures (PTSS, perceived disability, pain intensity and depressed mood) after controlling for demographics variables. Findings are expected to contribute to understanding of risk factors and prevention/intervention strategies for physical and psychosocial outcomes following SCI.

\section{MATERIALS AND METHODS}

\section{Participants}

A total of 55 participants (36 males) were recruited from a free standing rehabilitation hospital in the Southwestern United States. Recruitment, utilizing a convenience sample, took place from January 2014 to June 2016. The mean age was 47.78 years $($ s.d. $=15.65$; range $20-79)$. Of the sample, 33 participants identified as White (60\%), 8 as African-American (15\%), 6 as Hispanic (11\%), 3 as Asian (5\%), 3 as Multiracial (5\%), 1 as Native Hawaiian (2\%) and 1 as Native American (2\%). The majority (89\%) of the sample graduated high school, $13 \%$ obtained a Bachelor's degree and 5\% obtained a graduate degree. All participants were 18 years of age or older and had sustained an SCI. Level of injury was as follows: 31 cervical, 17 thoracic, 2 lumbar and 1 sacral (4 participants had missing data). ASIA Impairment Scale (AIS) scores were as follows: 13 complete injuries, 33 incomplete injuries and 9 had missing data. Inclusion criteria consisted of the following (a) the presence of recent SCI; (b) traumatic or non-traumatic (for example, infection and disk degeneration) SCI; (c) age 18 years or older; and (d) medical stability as determined by the attending physician. Patients with severe cognitive impairment or pre-morbid mental illness/developmental disability were excluded from recruitment. Upon admission to acute inpatient rehabilitation, participants who met recruitment criteria provided informed consent and subsequently enrolled in the study. Outcome measures were administered at the time of enrollment or before the participant was discharged. Not all participants received all of the measures, thus contributing to the missing data. The number of participants who completed each measure is listed in Table 1. Data from the current sample were included in a previously published paper regarding association between injustice appraisal, attribution of blame and intention to litigate ${ }^{26}$ and another paper on injustice appraisal and anger. ${ }^{27}$

\section{Measures}

Event centrality. Event centrality was assessed with the Centrality of Events Scale. ${ }^{18}$ The measure consists of seven items rated on a scale from 1 (totally disagree) to 7 (totally agree). Example items include 'I feel that this event has become part of my identity' and 'this event has become a reference point for the way I understand myself and the world'. We altered the instructions to specifically refer to their SCI (for example, 'I feel that this injury has become a part of my identity'). In the current sample, the internal reliability was $\alpha=0.85$.

Posttraumatic stress symptoms. The Primary Care Screen ${ }^{28}$ was used to measure PTSS. This measure consists of four items designed to screen for PTSD in medical settings. Participants were asked to refer to their SCI when 
Table 2 Multiple regression analyses predicting the four outcome variables-PTSD symptoms, perceived disability, pain intensity and depressed mood

\begin{tabular}{|c|c|c|c|c|}
\hline & $\mathrm{R}^{2}$ change & $F$ & $\beta$ & $t$ \\
\hline \multicolumn{5}{|c|}{ PTSD symptoms (PC-PTSD) } \\
\hline Step 1 & 0.07 & 3.42 & & \\
\hline Education & & & -0.23 & 1.75 \\
\hline Step 2 & 0.06 & 3.07 & & \\
\hline Depressed mood & & & 0.19 & 1.43 \\
\hline Step 3 & 0.07 & $4.15^{*}$ & & \\
\hline Event centrality & & & 0.27 & $2.04 *$ \\
\hline \multicolumn{5}{|l|}{ Perceived disability } \\
\hline Step 1 & 0.21 & $11.91^{* *}$ & & \\
\hline Depressed mood & & & 0.39 & $3.15^{*}$ \\
\hline Step 2 & 0.15 & $12.20^{* *}$ & & \\
\hline Event centrality & & & 0.39 & $3.18^{* *}$ \\
\hline \multicolumn{5}{|c|}{ Pain intensity (MPQ-SF-PPI) } \\
\hline Step 1 & 0.09 & $4.48^{*}$ & & \\
\hline Gender & & & 0.22 & 1.53 \\
\hline Step 2 & 0.06 & 3.42 & & \\
\hline Depressed mood & & & 0.27 & 1.85 \\
\hline \multicolumn{5}{|c|}{ Depressed mood (PHQ-8) } \\
\hline Step 1 & 0.09 & $4.42^{*}$ & & \\
\hline Gender & & & 0.22 & 1.51 \\
\hline Step 2 & 0.07 & 3.42 & & 1.85 \\
\hline Pain & & & 0.27 & \\
\hline
\end{tabular}

Abbreviations: MPQ-SF-PPI, McGill Pain Questionnaire-Short Form Present Pain Intensity Index; PC-PTSD, Primary Care PTSD screen; PHQ-8, Patient Health Questionnaire-8 item;

PTSD posttraumatic stress disorder.

$N=55, * P<0.05, * * P<0.01$

completing this measure. In the current sample, the internal reliability was $\alpha=0.72$.

Perceived disability. Perceived disability was assessed with the Pain Disability Scale. ${ }^{29}$ We altered the instructions from asking participants to 'measure the degree to which you think aspects of your life may be disrupted by pain' to 'measure the degree to which you think aspects of your life may be disrupted by your injury'. The measure consists of seven items rated on a scale of 0 (no disability) to 10 (worst disability). The measure asks participants to rate the extent of their disability in the areas of family/home, recreation, social activity, occupation, sexual behavior, self-care and life-support activities. In the current sample, the internal reliability was $\alpha=0.80$.

Pain intensity. To measure pain intensity, we used a numeric rating scale (NRS), with zero representing 'no pain' and 10 representing 'worst possible pain'reported on average over the past 2 weeks. The numerical ratings scale is supported as a valid and reliable measure of pain intensity. ${ }^{30}$

Depressed mood. We used the Personal Health Questionnaire Depression Scale-8 (PHQ- 8$)^{31}$ to measure depressed mood over the past 2 weeks. The measure consists of eight items rated on a scale from 0 (not at all) to 3 (nearly everyday). Example items include 'little interest or pleasure in doing things' and 'feeling tired or having little energy'. In the current sample, the internal reliability was $\alpha=0.76$.

\section{Procedure}

This study reports baseline data from a larger, ongoing longitudinal study that contains some measures not reported here. All procedures were approved by the appropriate Institutional Review Board.
Analyses

The data analytic plan began with descriptive statistics to examine the extent to which event centrality was prevalent in the sample of individuals with a SCI. We next examined zero-order correlations between event centrality scores and scores on the SCI-related outcomes of PTSS, perceived disability, perceived pain and depressed mood, along with demographic variables. Finally, we conducted hierarchical regression analyses to examine whether event centrality predicts unique variance in the SCI-related outcomes. Hierarchical regression was chosen so we could examine the predictive validity of event centrality after key covariates were entered into the model. We began each regression model by entering any demographic variables that evidenced a significant zero-order correlation with the outcome variable. We next entered any other variables that evidenced a significant zero-order correlation with the outcome variable. We entered event centrality last to test whether this variable could account for unique variance in the outcome variables. For the model predicting PTSS, given our sample size and number of predictors, we had 0.80 power to detect effect sizes of $f^{2}$ of 0.21 or higher $(0.15$ is considered medium and 0.35 is considered large). For the other three models (which included fewer predictor variables), we had 0.80 power to detect effect sizes of 0.19 or higher. Hence, we had adequate statistical power to detect medium or high effect sizes.

\section{RESULTS}

First, the extent to which the participants rated their injury as central to their identity was examined. As can be seen in Table 1, the average score on the Centrality of Events Scale was $M=22.86$, s.d. $=7.60$, with a possible range of $7-35$. This mean score is similar to mean scores on this scale reported for other traumatic events including undergraduate students who experienced a DSM-IV traumatic event $(M=24.92),{ }^{14}$ older adults $(M=20.00),{ }^{19}$ patients with social anxiety $(M=23.1)$ and patients with panic disorder $(\mathrm{M}=21.7) .{ }^{32}$ Perhaps most relevant, the mean score in this study is very similar to the mean score obtained in a sample of chronic pain patients ${ }^{25}(\mathrm{M}=68.9$ using the 20 -item version, which translates to $M=24.1$ if the seven-item version was used, which was used in this study and the aforementioned studies). The mean score obtained in this study is at the upper end of the possible range of scores and 3 s.d. covers the entire possible range of the scale. Hence, for the SCI patients in this study, construing their medical condition as central to their identity was commonplace, and there was ample variance in these scores.

The zero-order Pearson correlations between all variables were examined and are depicted in Table 1. Event centrality was significantly related to perceived disability and PTSS in the expected directions. However, event centrality was not significantly related to pain intensity or depressed mood.

\section{Regression analyses}

Next, hierarchical regression models were created to examine the relationships between event centrality and the four outcome measures after controlling for covariates. Only predictor variables that evidenced significant zero-order correlations with the outcome variable were included in the model. Table 2 shows the final model for each outcome variable.

Posttraumatic stress. Education level and depressed mood accounted for $13 \%$ of the variance in PTSS in the first two steps of the regression model (see Table 2). Importantly, when event centrality was added in the third step, the change in $R^{2}$ was significant, with event centrality uniquely accounting for an additional 7\% of the variance in PTSS.

Perceived disability. Depressed mood accounted for $21 \%$ of the variance in perceived disability in the first step. Importantly, when event centrality was added in the second step, the change in $R^{2}$ was 
significant, with event centrality uniquely accounting for an additional $15 \%$ of the variance in perceived disability.

Pain intensity. Gender accounted for 9\% of the variance in pain intensity in the first step, with females evidencing higher risk for pain intensity. Depressed mood accounted for an additional $6 \%$ of variance in the second step, but this was not a statistically significant increase in variance accounted for. Event centrality was not included in the regression model as it failed to evidence a significant correlation with pain intensity in Table 1 .

Depressed mood. Gender accounted for $9 \%$ of the variance in depressed mood in the first step, with females evidencing higher risk for depression. Pain intensity accounted for an additional $7 \%$ of variance in the second step, but this was not a statistically significant increase in variance accounted for. Event centrality was not included in the regression model as it failed to evidence a significant correlation with depressed mood in Table 1.

\section{DISCUSSION}

When an individual suffers a severe SCI, the patient faces very difficult physical challenges and much of the rehabilitation process focuses on physical recovery. However, it would be a mistake to ignore the perhaps equally as difficult psychological challenges the patient faces. PTSS and suicide are common in this population, ${ }^{12,33}$ although there is a large amount of variance in the extent to which SCI patients struggle with the psychological challenges. ${ }^{34}$ The present findings suggests new ways of identifying individuals who may be most at risk.

This was the first known study to examine event centrality in a sample of spinal cord patients. We found that the mean score for event centrality in our sample was similar to mean scores obtained for psychological traumas. There was ample variance in event centrality scores, suggesting spinal cord patients vary greatly in the extent to which they construe their injury as a central aspect of their identity and life story. Of note, participants completed the assessment measures at various points during their time in the rehabilitation center (but before discharge). The length of stay in rehabilitation and the extent to which goals were met or not met during this process can potentially affect levels of event centrality and other perceptions of SCI adjustment. Such factors likely contributed to the variability we observed in our participant sample. Importantly, we found that event centrality was significantly correlated with PTSS and perceived disability. Event centrality continued to significantly predict these two outcomes after controlling for demographics and depressed mood. These finding suggest event centrality accounts for unique variance in these outcomes. Event centrality failed to significantly correlate with our other two outcome measures-pain intensity and depressed mood, which were only predicted by gender, with females evidencing greater risk.

This study contains several limitations that are important to note. The first limitation is the limited sample size. When conducting multiple regression analyses, a sample of 55 participants limits the amount of available statistical power. A second limitation is the cross-sectional design. The data were collected on participants within the first 2 weeks of their admittance to a rehabilitation center. The trajectory of PTSS resulting from traumatic experiences vary greatly in the months following such events. ${ }^{32}$ Longitudinal studies are needed to examine the extent to which event centrality predicts the trajectory of PTSS in the months following injury. Finally, when completing the measure of event centrality, participants were asked to refer to their injury, which could be construed as either referring to the event that caused the injury, or the resulting physical impairments and being a SCI patient. We believe most participants construed the event as the latter, but future studies should be more explicit.

In the psychological trauma literature, event centrality has proven to be a consistently strong predictor of PTSS. ${ }^{13,14,21}$ In this study, we found event centrality is not only common among SCI patients, it also predicts unique variance in two important psychological outcomesPTSS and perceived disability. Numerous studies have found that patient perceptions and construals of their SCI is related to subsequent psychological outcomes. ${ }^{35,36}$ The current finding that construals of how central the injury has become to identity adds to this literature by highlighting the importance of event centrality. Given that subjective perceptions and construals have an important role in psychological outcomes, it is important to consider psychological treatments that affect such perceptions. Numerous studies have found that cognitive behavioral therapies are effective at altering toxic perceptions to healthier perceptions and relieving some of the psychological problems associated with pain. ${ }^{37}$ In reference to the specific perception of event centrality, two studies have found acceptance and commitment therapy (a type of cognitive behavioral therapy) can reduce event centrality. ${ }^{38,39}$ We hope that the results of this study will alert health-care professionals working with SCI patients as to the importance of event centrality as a possible risk factor for psychological difficulties in this population.

\section{DATA ARCHIVING}

There were no data to deposit.

\section{CONFLICT OF INTEREST}

The authors declare no conflict of interest.

1 Bryce TN, Biering-Sørensen F, Finnerup NB, Cardenas DD, Defrin R, Lundeberg T et al. International Spinal Cord Injury Pain Classification: part I. Background and description. Spinal Cord 2012; 50: 413-417.

2 Wollaars MM, Post MWM, van Asbeck FWA, Brand N. Spinal cord injury pain: the influence of psychologic factors and impact on quality of life. Clin J Pain 2007; 23: 383-391.

3 Agar E, Kennedy P, King NS. The role of negative cognitive appraisals in PTSD symptoms following spinal cord injuries. Behav Cognitive Psychother 2006; 34: 437-452.

4 Chung MC, Preveza E, Papandreou K, Prevezas N. The relationship between posttraumatic stress disorder following spinal cord injury and locus of control. J Affect Disord 2006; 93: 229-232.

5 Kennedy P, Evans MJ. Evaluation of post-traumatic distress in the first 6 months following SCl. Spinal Cord 2001; 39: 381-386.

6 Krause JS, Saunders LL, Newman S. Posttraumatic stress disorder and spinal cord injury. Arch Phys Med Rehabil 2010; 91: 1182-1187.

7 Nielsen MS. Post-traumatic stress disorder and emotional distress in persons with spinal cord lesion. Spinal Cord 2003; 41: 296-302.

8 Schönenberg M, Reimitz M, Jusyte A, Maier D, Badke A, Hautzinger M. Depression, posttraumatic stress, and risk factors following spinal cord injury. Int J Behav Med 2014; 21: 169-176.

9 Craig A, Perry KN, Guest R, Tran Y, Dezarnaulds A, Hales A et al. Prospective study of the occurrence of psychological disorders and comorbidities after spinal cord injury. Arch Phys Med Rehabil 2015; 96: 1426-1434.

10 Otis C, Marchand A, Courtois F. Risk factors for posttraumatic stress disorder in persons with spinal cord injury. Top Spinal Cord Inj Rehabil 2012; 18: 253-263.

11 American Psychiatric Association. Diagnostic and Statistical Manual of Mental Disorders, 5th edn. American Psychiatric Association: Washington, DC, USA. 2013.

12 Chung MC, Preveza E, Papandreou K, Prevezas N. Locus of control among spinal cord injury patients with different levels of post-traumatic stress disorder. Psychiatry Res 2007; 152: 253-260.

13 Berntsen D, Rubin DC. When a trauma becomes a key to identity: enhanced integration of trauma memories predicts posttraumatic stress disorder symptoms. App/ Cognitive Psychol 2007; 21: 417-431.

14 Rubin DC, Boals A, Hoyle RH. Narrative centrality and negative affectivity: independent and interactive contributors to stress reactions. J Exp Psychol Gen 2014; 143: 1159-1170.

15 Brown A, Antonius D, Kramer M, Root J, Hirst W. Trauma centrality and PTSD in veterans returning from Iraq and Afghanistan. J Trauma Stress 2010; 23: 496-499. 
16 Barton S, Boals A, Knowles L. Thinking about trauma: the unique contributions of event centrality and posttraumatic cognitions in predicting PTSD and posttraumatic growth. J Trauma Stress 2013; 26: 718-726.

17 Robinaugh DJ, McNally RJ. Trauma centrality and PTSD symptom severity in adult survivors of childhood sexual abuse. J Trauma Stress 2011; 24: 483-486.

18 Berntsen D, Rubin DC. The centrality of event scale: a measure of integrating a trauma into one's identity and its relation to post-traumatic stress disorder symptoms. Behav Res Ther 2006; 44: 219-231.

19 Boals A, Hayslip B Jr, Knowles LR, Banks JB. Perceiving a negative event as central to one's identity partially mediates age differences in posttraumatic stress disorder symptoms. J Aging Health 2012; 24: 459-474.

20 Schuettler D, Boals A. The path to posttraumatic growth versus PTSD: contributions of event centrality and coping. J Loss Trauma 2011; 16: 180-194.

21 Fitzgerald JM, Berntsen D, Broadbridge CL. The influences of event centrality in memory models of PTSD. App/ Cognitive Psychol 2016; 30: 10-21.

22 Blix I, Birkeland MS, Solberg $\varnothing$, Hansen MB, Heir T. The launching and ensnaring effects of construing a traumatic event as central to one's identity and life story. App Cognitive Psychol 2016; 30: 526-531.

23 Boals A, Ruggero C. Effects of narrative centrality on PTSD symptoms: evidence from a prospective and longitudinal design. Anxiety Stress Coping 2016; 29: 533-541.

24 Boelen PA. A prospective examination of the association between the centrality of a loss and post-loss psychopathology. J Affect Disord 2012; 137: 117-124.

25 Perri LM, Keefe FJ. Applying centrality of event to persistent pain: a preliminary view. J Pain 2008; 9: 265-271.

26 Trost Z, Monden KR, Buelow M, Boals A, Scott W. Perceived injustice predicts intention to litigate: findings from a spinal cord injury sample. Psychol Injury Law 2016; 9: 31-40.

27 Trost Z, Scott W, Buelow M, Nowlin L, Turan B, Boals A et al. The association between injustice perception and psychological outcomes in an inpatient spinal cord injury sample: the mediating effects of anger. Spinal Cord 2017; 55: 898-905.
28 Prins A, Ouimette P, Kimerling R, Cameron RP, Hugelshofer DS, Shaw-Hegwer J et al. The primary care PTSD screen (PC-PTSD): development and operating characteristics (PDF). Primary Care Psychiatry 2003; 9: 9-14.

29 Downie WW, Leatham PA, Rhind VM, Wright V, Branco JA, Anderson JA. Studies with pain rating scales. Ann Rheum Dis 1978; 37: 378-381.

30 Tait RC, Chibnall JT, Krause S. The pain disability index: psychometric properties. Pain 1990; 40: 171-182.

31 Kroenke K, Strine TW, Spritzer RL, Williams JB, Berry JT, Mokdad AH. The PHQ-8 as a measure of current depression in the general population. J Affect Disord 2009; 114: $163-173$.

32 O'Toole MS, Watson LA, Rosenberg NK, Berntsen D. Negative autobiographical memories in social anxiety disorder: a comparison with panic disorder and healthy controls. J Behav Ther Exp Psychiatry 2016; 50: 223-230.

33 Soden RJ, Walsh J, Middleton JW, Craven ML, Rutkowski SB, Yeo JD. Causes of death after spinal cord injury. Spinal Cord 2000; 38: 604-610.

34 Bonanno GA. Loss, trauma, and human resilience: have we underestimated the human capacity to thrive after extremely aversive events? Am Psychol 2004; 59: 20-28.

35 Krause JS, Edles PA. Injury perceptions, hope for recovery, and psychological status after spinal cord injury. Rehabil Psychol 2014; 59: 176-182.

36 Middleton J, Tran Y, Craig A. Relationship between quality of life and self-efficacy in persons with spinal cord injuries. Arch Phys Med Rehabil 2007; 88: 1643-1648.

37 Jungquist CR, Tra Y, Smith MT, Pigeon WR, Matteson-Rusby S, Xia Y et al. The durability of cognitive behavioral therapy for insomnia in patients with chronic pain. Sleep Disord 2012; 2012: 679648.

38 Boals A, Murrell AR. I am > trauma: experimentally reducing event centrality and PTSD symptoms in a clinical trial. J Loss Trauma 2016; 21: 471-483.

39 Boals A, Murrell AR, Berntsen D, Southard-Dobbs S, Agtarap S. Experimentally reducing event centrality using a modified expressive writing intervention. $J$ Context Behav Sci 2015; 4: 269-276. 